\title{
Simulation of single-ventricle physiology ... a start
}

\author{
M'hamed (Hamy) Temkit, $\mathrm{PhD},{ }^{\mathrm{a}}$ and Tara Karamlou, $\mathrm{MD}^{\mathrm{b}}$
}

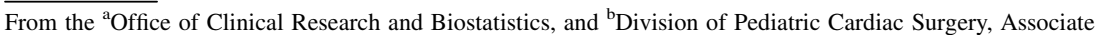
Professor of Surgery, Mayo Clinic and Phoenix Children's Hospital, Phoenix, Ariz. Disclosures: Authors have nothing to disclose with regard to commercial support.

Received for publication Oct 1, 2017; accepted for publication Oct 6, 2017; available ahead of print Nov 2, 2017. Address for reprints: Tara Karamlou, MD, Mayo Clinic and Phoenix Children's Hospital, 1919 E Thomas Rd, Heart Center, Main Building, Phoenix, AZ 85016 (E-mail: tkaramlou@phoenixchildrens.com).

J Thorac Cardiovasc Surg 2018;155:722-3

$0022-5223 / \$ 36.00$

Copyright $(c) 2017$ by The American Association for Thoracic Surgery

https://doi.org/10.1016/j.jtcvs.2017.10.023
}

In this issue of the Journal, Conover and colleagues ${ }^{1}$ introduce their concept of "An Interactive Simulation Tool for Patient-Specific Clinical Decision Support in Single Ventricle Physiology." The authors have developed a beta version for an interactive tool based on the lumped parameter models (LPMs) to estimate the hemodynamic (physiologic) impact of medical and surgical interventions during all 3 stages of single-ventricle palliation. The use of the LPM approach where the circulation is divided into several interconnecting compartments reduces the time needed to produce the simulation results compared with that of the more detailed local hemodynamic information of the computational fluid dynamics mathematic models. To assess the accuracy of the LPM approach, the authors collected data from 60 total cardiac catheterization reports (20 from each of the 3 stages of single-ventricle palliation) from 47 subjects. Cardiac catheterization measurements were not obtained at a standardized time in relationship to the staged surgical procedure, and it is not clear what methods were used to assess the validity or veracity of the catheterization parameters. The catheterization results were then compared with simulator outputs by using statistical and visual tools, such as the paired $t$ test, simple linear regression, and Bland-Altman analysis. Specifically, 4 simulator outputs were compared with catheterization findings: pulmonary to systemic flow ratio (Qp:Qs), systemic arterial saturation ( $\mathrm{SaO} 2)$, mean pulmonary arterial pressure, and systemic-venous oxygen difference (SaO2$\mathrm{SvO} 2$ ). On the basis of the results presented, the simulator outputs generally agreed with those of the cardiac catheterization results, with the exception of the average $\mathrm{SaO} 2-$ $\mathrm{SvO} 2$, where there was a significant difference of $1 \%$.

The simulator described in the present report is based on previous excellent work by this group to evolve the field of computational fluid dynamics by integrating LPM into a clinical construct. ${ }^{2}$ Clearly, Conover and colleagues ${ }^{1}$ have made an important first step with the development of this simulator and the forthcoming web-based application, which may be used by both clinicians and families. This work will facilitate incorporation and increase accessibility of this complex technology into mainstream clinical arenas.

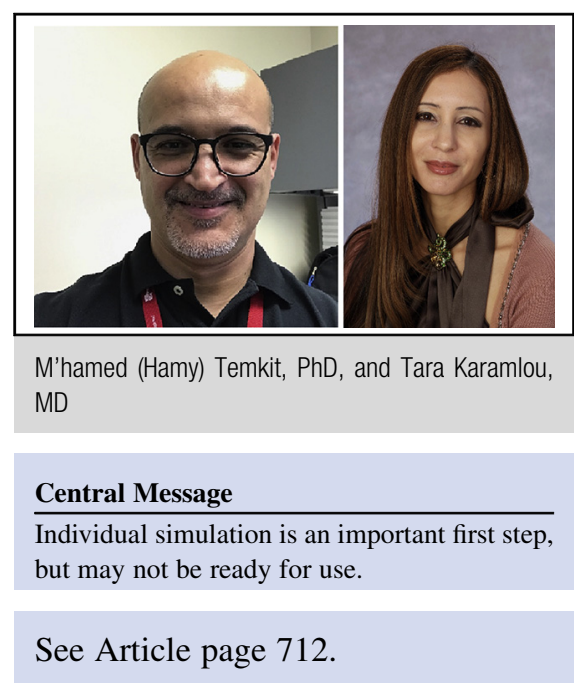

However, despite these innovations, I have a few comments about the relevance of this tool as currently described and elucidate some important limitations to consider. I acknowledge that further iterations could potentially obviate some of these limitations. The first limitation is partly due to the restrictions imposed by current clinical practice, because most patients do not undergo postsurgical or interventional repeat catheterization to assess the impact of such interventions. Thus, the simulated outputs for modifications in terms of moving from one surgical stage to another lack validation of the immediate modified state, because the outputs are not compared with catheter-based hemodynamics in the new state. In other words, hemodynamic effects of virtual surgery to convert a patient in stage 1 with a set of characteristics to stage 2 cannot be validated immediately because post-Glenn catheterization data were not available. It is true that the pre-Fontan hemodynamics can be, and were in this study, substituted, but implying correlations or causal relationships from measurements obtained several years apart is a stretch. It might be useful for the authors to have commented on whether magnetic resonance imaging data or other surrogates (ie, intensive care unit data from invasive lines) could be used or incorporated in lieu of the cardiac catheterization data to validate the simulated outputs within a more plausible time frame.

It is also unclear to me how the authors integrated the cardiac catheterization reports from the 47 patients. Ostensibly, some were used more than once (which could imply enhanced correlation between some of the measures that were not accounted for statistically), but this is not clear from the current article. Further, if some cardiac catheterizations were obtained during a period of marginal stability, 
when multiple interventions might be performed in a patient simultaneously, did this influence the agreement between the simulated outputs and the measured data? I would encourage the authors to provide some context and timing around the cardiac catheterization data used to validate the simulator outputs.

The second potential limitation is that the current simulator cannot account for certain important physiologic variables, such as neoaortic valve or atrioventricular valve insufficiency in single-ventricle states. Consequences of volume loading of the systemic single ventricle would be critical to incorporate into any clinical decisionmaking about the downstream consequences of discrete interventions, and therefore the inability to simulate the impact of these lesions reduces the utility of this technology.

I am also confused as to the reason why the authors avoided using Bayesian inferences and methods to reduce variance and enhance model performance. This group has expertise with these methods and used it successfully in their initial description of LPM for patients with singleventricle physiology. ${ }^{2}$ Given that the current estimates (as seen in the Bland-Altman plots) had significant variance, use of Bayesian priors might be a worthwhile exercise. At present, this variability is attributed to the inherent variability of some catheter-derived measurements and the imprecision of others. In truth, this seems a bit of an empty excuse and could have been easily avoided or mitigated by collecting prospective standardized measurements, repeating them, and using an average. The use of a prospective design to validate this novel simulator would also avoid the issues of timing.

Finally, the tool is not presently suited to predict many extremely common medical interventions among patients with single-ventricle physiology, such as the impact of initiation of milrinone therapy or blood transfusion, because of the complexity of the impact of such therapies. In other words, until we know all of the unknowns, how can we simulate the outcome of such interventions? I would argue that the most challenging decision-making dilemmas occur precisely when a patient behaves in a physiologic manner that is unexpected given the baseline data. Many of us have encountered a patient who had suitable hemodynamics at the pre-Fontan catheterization, yet eventuated in an unsuccessful Fontan circulation, or the patient post-Glenn who is hypoxemic despite normal hemodynamic data. Moreover, because individuals respond differently, a given set of parameters may not predict success or failure of an intervention. It is an unfortunate fact that the incontrovertible individuality of each patient dictates that a response is unpredictable and therefore may obviate even the most sophisticated attempt at simulation. A powerful next step for this group would be to harness the data from the simulators in a prospective study and correlate them with a meaningful clinical outcome.

Several points can be made regarding the use of these statistical methods. In their revised Table 2, the authors should also provide the standard deviation along with the mean difference so that the reader can both assess the variability and compute the effect size and power of the paired $t$ test. This is especially relevant given that only 20 catheterization reports are available at each stage. The authors also should consider a more appropriate measure of agreement between the catheterization results and the simulated results, such as the intraclass correlation instead of the coefficient of variation (R2) from the linear regression and the Bland-Altman analysis, which is only a visualization method.

In my mind, it is likely that at present, this tool is most suited as an educational tool for learners and families. The video submitted by the authors is a highly effective and useful tool that could enhance bedside teaching and augment discussions with families about the risks and benefits (in a general sense) of different options. I would reserve assumptions about the clinical utility of the simulator until some of the limitations can be overcome... and I look forward to a prospective study from this dedicated group of investigators.

\section{References}

1. Conover T, Hlavacek AM, Migliavacca F, et al. An interactive simulation tool for patient-specific clinical decision support in single ventricle physiology. J Thorac Cardiovasc Surg. 2018;155:712-21.

2. Schiavacci DE, Baretta A, Pennati G, Hsia TY, Marsden AL. Patient-specific parameter estimation in single-ventricle lumped parameter circulation models under uncertainty. Int J Numer Methods Biomed Eng. 2017;33:2799. 